\title{
Retraction Note to: Consequences of Binary Chemically Reactive Flow Configuration of Williamson Fluid with Entropy Optimization and Activation Energy
}

\author{
M. ljaz Khan ${ }^{1} \cdot$ A. Alsaedi ${ }^{2}$ Sumaira Qayyum ${ }^{1} \cdot$ T. Hayat $^{1,2}$
}

Published online: 14 September 2021

๑) Springer Science+Business Media, LLC, part of Springer Nature 2021

\section{Retraction to: International Journal of Thermophysics (2019) 40:94 https://doi.org/10.1007/s10765-019-2563-8}

The Editor-in-Chief has retracted this article. After publication, a reader raised, among others, the following concerns:

1. There is a term RD missing from the dimensional Eqs. 1-5, This term is also absent from the transformed Eqs. 6-10, while it appears suddenly in Eq. 14.

2. There is no explanation why the numerical results do not satisfy the correct boundary conditions $(\theta(\eta=0)=\varphi(\eta=0)=1)$.

3. The authors provided no comment regarding the insufficient truncation of the computational domain.

Post-publication peer-review found these errors to undermine the article. The Editor-in-Chief no longer has confidence in the reliability of the work presented. The authors do not agree to this retraction.

Publisher's Note Springer Nature remains neutral with regard to jurisdictional claims in published maps and institutional affiliations.

The original article can be found online at https://doi.org/10.1007/s10765-019-2563-8.

M. Ijaz Khan

mikhan@math.qau.edu.pk; ijazfmg_khan@yahoo.com

1 Department of Mathematics, Quaid-I-Azam University, Islamabad 44000, Pakistan

2 Nonlinear Analysis and Applied Mathematics (NAAM) Research Group, Department of Mathematics, Faculty of Science, King Abdulaziz University, P.O.Box 80257, Jeddah 21589, Saudi Arabia 